\title{
Paul Farellier aux solstices
}

\author{
Monique W. Labidoire \\ Rueil-Malmaison, France
}

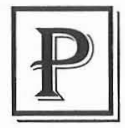

aul Farellier saisit la lumière mais aussi l'ombre sur de larges étendues, puis les resserre en quelque méditation intérieure. Cela conduit à un moment de respiration poétique tout à fait singulier.

Le poète cherche un éclairage au monde mais aussi un éclairage à sa propre existence. Il se peut que la connaissance de soi-même conduise à mieux appréhender les autres et permette le partage d'une totalité qu'on ne peut pas toujours nommer.

Quatre thèmes apparaissent principalement dans cette ouvre : la lumière, l'obscur, le silence et la durée, des thèmes, qui parcourent l'œuvre et interferent de manière récurrente. Le lexique choisi par Paul Farellier éclaire l'objet de l'œuvre et l'on peut y relever par exemple des mots comme, ruines, cendres, limites, fenêtres, demeure ou château et d'autres séries concordantes qui donnent leur sens au poème. Essentiellement, le poète se trouve aux solstices, aux solstices, car la distance qui le sépare ou le rapproche du centre de lui-même peut varier selon les saisons, les lieux, l'approche du poème et toutes postures plus intimes de réflexion, de méditation, d'interrogation sur le monde et sur le poème.

On apprend beaucoup sur un poète en respectant l'ordre chronologique des parutions même si ce n'est pas celui de l'écriture. Et regardant le premier recueil de Paul Farellier publié en 1984 au Pont de l'Epée, chez Guy Chambelland, il s'intitule L'intempérie douce une certaine abstraction est visible en écho à une retenue, particulièrement dans la première partie intitulée "En ce qui reste d'été ". Un peu abstraite, cette

* Cet article est tiré d'un exposé fait par MWL au " Mercredi du Poète " le 25 janvier 2006. Les réponses de Paul Farellier sont reproduites ici avec son accord. 
poésie semble garder une distance avec son locuteur dans ce sens où il désire vivre le poème et la poésie, sans pour autant se montrer de plein fouet dans son propre champ poétique. Mais une lecture attentive favorise une approche plus précise et offre quelques signes auxquels le lecteur peut s'accrocher. Le poète est lui-même dans une vastitude, et du centre au solstice, il y a place pour le mystère et l'inconnu. Il écrit : "Quelque chose qui n'est pas encore en ordre, se dispose lentement: un semoir invisible au fond de l'air"

Les années 1980 sont une période de la poésie contemporaine où le lyrisme semble pas mal jeté aux orties et si Paul Farellier s'y livre au lyrisme, c'est entre les lignes et dans le blanc du poème à des degrés qu'il faut déchiffrer patiemment. Il s'exclame : "Quelle retenue dans l'écriture de la beauté!" Mais il lui plaît aussi de se livrer quand il dit : "Ce cour, tu le retiens pour plus largelpour y bercer le plus vaste ». C'est dit, le cœur de Paul Farellier ne se contentera pas d'un petit air de musique! Et si les émotions et les sentiments sont fortement pressentis, le poème garde ses limites et contient tout débordement. Pourtant l'émotion que délivrent les poèmes permet d'approcher une épaisseur dans laquelle s'établit un accueil, ce qui est à l'opposé d'une mise à l'écart du poète dans une quelconque tour d'ivoire. Le poète cherche un passage qu'il pourrait pratiquer avec d'autres. Mais de quel passage s'agit-il, de celui qui pourrait s'ouvrir entre lumière et obscur, entre silence et solitude, entre beauté et durée et qui rejoindrait une communauté, un partage? Espoirs confondus de trouver un jour un sens à l'existence, à la sienne propre comme à celle du monde? Et il nous dit : " $C e$ qui fait la question durer, c'est que le passage existe".

L'homme garderait-il en lui une blessure endémique incurable qui ne pourrait se soulager que par toutes ces questions et sans pouvoir y répondre? Le poète semble accepter cet état dont il n'est pas maître. Il se trouve "Dans la nuit passante ". Le passage est bien visible, mais il y fait nuit. Il semble espérer une grâce équitable qui dépasserait la nuit.

Une expérience qui va l'amener à observer, écouter, ressentir et entrer dans une réalité, qui si elle n'est pas encore complètement compréhensible, chemine au milieu d'instant vécus où le ciel, la rose, le talus, convoquent, à une heure dans laquelle la nuit obscurcit les étoiles, d'étranges convives qui vont poser, - se poser la question fondamentale -, au moins pour un poète : avec quoi nourrir le poème et l'existence? Sur quelle étendue poser le poème? Ne doit-on pas recourir à la nudité, à l'espace désencombré de toutes les scories qui s'entassent dans les mémoires, s'alléger et prendre son envol vers la transparence, accueillir le poème sans bien savoir d'où il vient et dire : 


\section{"La table de ce côté}

\section{Le couvert pour traiter l'ombre}

Nous dînerons d'une pensée déchirante "

Le poète semble dire qu'il faut se mettre à table et redécouvrir cet espace pour mieux partager le pain et le vin. À condition de ne pas les servir flétris, les mots nourriront les convives de ce banquet. Qu'entendons-nous dans ces trois vers? Nous entendons que la parole peut élargir la déchirure, que la conscience de l'absence, de la durée, de la mort n'est pas vouée au désespoir mais peut être acceptée. Il y a donc questionnement intime et conscient. Il y a aussi questionnement sur ce qui entoure. Il faut chercher un passage entre le dehors et le dedans mais trouver aussi le paysage et le lieu. Écouter et ressentir, toucher le sentiment qui élève vers quelque chose qui est toujours pour le poète, de l'ordre de l'inconnu et qu'il désire ardemment rencontrer.

Pour y parvenir, le poète ne va pas se contenter de se poser des questions afin de résoudre une énigme, il ne peut se confiner dans des espaces réduits au regard de soi-même, il peut, il doit, tout simplement respirer la fleur du talus et être attentif à "L'été sur sa perte »,.. il peut aussi travailler en direct sur le motif à l'exemple de ces vers qui ouvrent un espace de paix :

\section{Il fait beau.}

De grandes distances nous pénètrent.

La rivière dessine, retarde le pays.

J'écoute vivre.

Ce poème dit bien ce qu'il veut dire. Nous sommes exactement dans le sujet du monde, dans la proximité d'un questionnement et d'un début de réponse apportée par le poète : "j'écoute vivre » ce qui ne veut pas dire qu'il ne fait qu'écouter sans participer, mais c'est par ce sens de l'écoute que son regard s'affûtera.

Paul Farellier ne traite pas ses motifs à la manière d'un peintre, mais plus symboliquement dans le creusement de la connaissance de la matière monde. Cette quête d'un espace plus habitable le conduit à dessiner un paysage poétique qui servira de support, voire de creuset au poème. $\mathrm{Ce}$ sont les mots et les thèmes qui construisent l'épaisseur du poème tandis que les motifs aussi suggestifs soient-ils n'en sont qu'une trame, mais une trame solide et nécessaire pour envelopper l'objet du poème. 
Monique W. Labidoire : La poésie moderne, contemporaine, n'est-elle pas devenue plus philosophique que poétique. Ne se questionne-t-elle pas plus sur le monde qu'elle nous le montre et qu'elle nous le dit? Et aussi : ne s'interroge-t-elle pas plus sur l'outil et le matériau poétiques qu'elle ne construit le poème?

Paul Farellier : Poésie et philosophie pourront-elles un jour échapper à leur fascination réciproque? Et d'ailleurs le faut-il? Les deux sont étroitement mêlés. Si le poète, à sa manière — qui rappelons-le ne peut pas être conceptuelle -, se tourne vers une approche spéculative, il ne fait que déférer à cette exigence, en effet bien contemporaine, qui le porte à juger la légitimité de sa parole face à la réalité du monde : il y a maintenant, sans nul doute, chez beaucoup de poètes, peut-être même avant le sentiment d'un rapport au monde, un souci prévalent du statut du langage poétique.

Ceci étant, la poésie peut tout perdre - et d'abord sa propre nature de poésie -à s'arroger une quelconque "mission" philosophique. Si elle doit être, à linstar de la philosophie et comme je le crois, une authentique manière de vivre au monde dans la recherche de sa propre vérité, elle ne reste poésie qu'en tenant fermement ses mots à l'écart des "Idées 》. Ne nous soucions pas de l'Idée. L'Idée saura briller d'elle-même aux yeux du philosophe quand il se penchera sur le poème spontané.

Vous posez le problème de ce quion a pu appeler "la poésie de laboratoire", celle du langage pour le langage, de l'autonomie aprement revendiquée du signifiant. Je dirai simplement que malgré ces recherches, purement intellectuelles, quelque chose a fait que nous n'avons jamais totalement perdu la poésie. On le doit à tous ceux qui ont maintenu, dans la diversité des écritures, une "vérité de parole ", pour employer une formule célèbre. Et cette vérité me semble toujours dériver de la leçon rilkéenne à savoir que la poésie authentique ne peut naître que de l'accumulation d'une expérience existentielle, qu'une poésie doit se vivre avant de sécrire. Qu'ont donc fait ceux qui ont suivi cette éthique? Ils ont gardé le lien avec une terre, une commune présence, la grâce du sensible. Ils ont gardé le sentiment du temps qui les a enseignés par leur attention à l'éphémère et au fragile. Et ils n'ont jamais rompu avec l'émotion, et surtout pas avec l'émotion-reine qu'est l'amour. Ainsi, un sens a-t-il été préservé, à travers lequel la poésie peut exister comme chance de salut pour ce monde.

La poésie est restée une chance de salut pour le monde, dit Paul Farellier qui est bien au solstice de la lumière éclairante comme connaissance d'un monde en constante mutation. Le poème peut-il sauvegarder le monde au 
moins dans le court instant de sa naissance si ce n'est pour une éternité toute relative? Peut-on l'espérer, ne serait-ce que dans ce fragment du temps?

Paul Farellier est au solstice de la durée temporelle, du temps qui passe, de cet espace-temps si difficilement saisissable, il est au plus près des saisons déclinantes qui s'élèvent au fil des mois de l'année, reflètent les saisons de la vie. Ainsi du titre de son recueil intitulé "Où la lumière s'abrège ». Une sorte de journal, de carnet de route, dans lequel chaque mois du calendrier invite au poème et dévoile un chemin sur lequel le poète vit son aventure. Une aventure qui peut, peut-être, tenir lieu de révélation. Un chemin de vie où des paysages sont proposés, des instants sont partagés, mais aussi où le destin de l'humanité dans sa fatalité incontournable est suggéré comme : "la morne répétition de lirréparable ".

Voici les premiers mois de l'année, des mois sombres pendant lesquels la lumière s'abrège. Le poète vit intensément cette perte du jour assimilé à la perte de quelque chose d'impalpable, ressenti intimement dans sa chair. La lumière est attendue, espérée mais pour le poète, le soir, le couchant, la nuit, l'obscur sont bien souvent du poème. Parvenu au mois de juin, au solstice de la Saint-Jean d'été, il s'interroge sur cette lumière revenue. "Qu'en ferons-nous maintenant, puisque nous n'en avons plus la pratique, nous qui sommes définitivement entrés dans la nuit, dans l'obscur. Que ferons-nous de : "cette lumière dont on n'a pas l'usage! "?

Monique W. Labidoire : Paul Farellier quelle est cette lumière dont on n'a pas l'usage? Et si nous n'avons pas l'usage de la lumière, nous faut-il rester dans l'obscur?

Paul Farellier : «cette lumière dont on n'a pas l'usage! ». Ici le poème porte un point d'exclamation. Il fait ressentir une sorte de scandale : l'expression ne serait-elle pas a priori monstrueuse? Elle le serait à coup sûr sìl s'agissait d'un déni de la lumière. Encore faut-il sinterroger sur le mot "usage ". S'agit-il de l'utilité, du droit d'usage, de la jouissance d'un bien dont on n'est pas propriétaire? Toute une polysémie surgit à l'analyse. Le poème enchaîne aussitôt par ce vers "L'usage s'est perdu des couchants dans la poussière ». Et donc, l'on entend bien que nous avons oublié un usage, une coutume, une tradition, la culture de cette lumière. Ici, le poème n'est pas un déni de la lumière mais éloge et célébration.

L'obscur ici ne soppose pas à la lumière; il en est le double et le miroir; le lieu d'élection et l'espace de rêve, de mémoire et de recréation. L'obscur, si l'on veut, comme creuset de lumière. 
L'astre éclairant, au plus haut de sa verticalité, appelle le poète à retrouver quelques fragments de sa mémoire et à les mettre au jour. Des lieux ont vécu qui ne sont plus mais qui restent gravés dans le poème. L'obscur et la lumière, la nuit et le jour sont alliés dans le destin humain : les hommes sont condamnés à une durée limitée dans laquelle s'inscrivent l'absence et la disparition. Comment, dans ces conditions, peut-on saisir la joie de tous les matins du monde? Le poète ouvre sa fenêtre et regarde. Entre autres saisons, le poète contemple l'été qui semble être vécu comme la saison la plus propice à la méditation. Il prend alors le goût et la saveur du monde, écoute les guêpes zigzaguer autour des confitures. Après tout, n'est-ce pas suffisant? se demande-t-il.

En septembre le soleil descend plus tôt dans la soirée et la mémoire du poète va s'inspirer du jour déclinant de la fin de l'été pour trouver sagesse et équilibre : Le jour va finir dans un soleil de raisin, écrit-il, s'offrant quelque respiration sur le motif d'un bonheur simple. Lumière et silence se confondent et, au fil des mois, "la lumière va croiser une nuit précoce ", elle va s'élancer sur des chemins plus ténébreux, plus silencieux comme l'homme dans le déclin de son âge. Jusqu'au solstice d'hiver où les feux s'éteignent, les feuilles ont disparu, craquelées, roussies. Plus de fleurs ni d'insectes, pas beaucoup d'oiseaux. Un pas vers la solitude. Un pas vers la disparition.

Ces pas, Paul Farellier les vit au centre des quatre éléments, à des degrés divers, mais dans une présence récurrente : terre, feu, air, eau apparaissent dans cette poésie construite dans l'épaisseur des matières déjà citées : la lumière, l'obscur, la durée, le silence, la solitude, la beauté qui ouvrent grand l'imaginaire. Les sens du "Voyant " sont dans un magma sensible, les textes sont sillonnés de la nature animale, végétale, minérale et humaine constituant le creusement du langage.

Les mots de Paul Farellier, en osmose avec les éléments et les sens, construisent un champ poétique très personnel où les jeux de lumières et de ténèbres, de connaissance et d'ignorance, du réel comme de l'imaginaire, s'alchimisent et se confrontent. Le poète cherche la clé d'un espace qu'il ne réussit pas toujours à saisir, une clé qui parfois se casse dans la serrure et laisse la porte fermée. Pour ouvrir cette porte, il lui faut peutêtre contourner son poème et le renverser.

" $A$ l'obscur et au vent ", justement; c'est le titre du recueil suivant et qui s'inscrit dans la continuité de la quête. Le passage à l'obscur n'est pas un constat d'échec, c'est la persévérance à trouver le lieu de l'écoute, à observer une fois encore avec une attention toujours plus aiguë les bruits 
du monde. Le poète s'interroge sur son observation; n'a-t-il pas perdu son temps à de trop petites choses? Et il écrit :

Au fond de toi-même, tu avises parfois quelque lieu sans repos, lieu de l'étonnement. À ne pas savoir t'y transporter dans linstant, tu te sentirais presque coupable de tranquillité; d'avoir étagé bien du temps sur des riens, manqué le chemin peut-être...

La persévérance sans doute, mais aussi la volonté de creuser toujours plus profond les thèmes. Cet obscur apparaît maintenant et rend plus de clarté à l'instant présent et plus compréhensibles les moments heureux dont la mémoire prend acte et diffuse, peu à peu, le paysage d'autres temps retrouvés. Il y a chez Paul Farellier une âme proustienne qui a besoin de prendre son temps pour retrouver sa mémoire et mettre en abyme des événements passés afin de les revivre dans le présent du poème. Le poète semble vivre dans une recherche, non pas d'un temps perdu, mais d'un temps dans lequel s'inscrirait une continuité, la mémoire constituant une entité dans laquelle s'uniraient passé, présent et une avancée possible vers le futur. Le temps retrouvé de Paul Farellier pourrait être le présent, ce présent vécu dans l'intensité du poème et qui apparaît comme une condition indispensable à son existence. L'instant du poème, multiplié par la durée et qui n'aurait ni commencement ni fin. "Un recoin du temps» où dit le poète "l'immobile s'y prolonge ". Mais comme Marcel Proust, Paul Farellier fait bouger quelque chose qui peut sembler immobile et qui pourtant change et vibre. S'arrêtant dans l'instant pour le mieux saisir et le contempler, il s'octroie la possibilité de cerner le motif, de l'étudier, de le respirer, d'en rendre compte.

Monique W. Labidoire : Paul Farellier je viens d'évoquer votre "âme proustienne ». Et l'on sait que du point de vue de la forme Marcel Proust adopte plutôt des phrases très longues. Comment expliquez-vous que pour vous, ce long mûrissement du poème, cette méditation, cette rumination aboutissent à une forme courte?

Paul Farellier : Laissez-moi dire d'abord mon étonnement initial et la très forte surprise que j'ai ressentie à ce rapprochement. Il m'honore, bien entendu, et à l'excès. Lecteur assidu de "la Recherche " je n'aurais pourtant pas eu l'audace de m'en réclamer. Mais à la réflexion, je dois admettre que, essentiel chez Proust, le jeu du temps et de la mémoire peut donner, pour nombre de mes 
écrits une certaine créance à la relation que vous avez bien voulu indiquer. Encore faut-il faire observer que d'autres aspects, des pans entiers de l'auvre proustienne - en particulier sa profondeur dans l'analyse psychologique - ne semblent induire aucune résonance dans mes fragments.

Et puisque ce mot "fragment "vient d'être prononcé, j'en viens à votre question très directe. Pourquoi cette forme courte? Je ne peux que la constater, non l'expliquer. Elle ne procède d'aucune décision, d'aucune stratégie. De même, elle ne traduit aucune pose, ni désir de paraître. La vérité c'est que je ne sais pas écrire "long".

C'est vrai que nous n'avons pas chez Paul Farellier de discours analytiques et psychologiques. Il s'agit plutôt de questionnement intérieur et de dialogues très concis avec les arbres, le vent, la nuit, avec, peut-être, une présence cachée que Pierrick de Chermont qui vient de consacrer une longue étude à l'œuvre du poète relève dans son chapitre intitulé « La foi inavouée ». Une présence qui serait de l'ordre du spirituel sinon du religieux. Oui, le poète habite le poème dans le silence d'une solitude qui est un appel. Le poème appelle à quelque chose qui tient du plus secret, de l'autrement, il est dans un ailleurs un peu flou qu'on peut nommer de diverses façons. Mais s'il est vrai que le poète écrit pour lui-même, pour mieux comprendre dans «quoi » il se trouve, «ce grand quoi » qui appelle le « comment » et le " pourquoi », il cherche aussi à nommer. Alors, certains nommeront Dieu, d'autres le Grand Architecte de l'Univers ou bien évoqueront une Présence; et d'autres encore suivront la piste du hasard et de la nécessité...

Paul Farellier ressent une présence au plus intime de sa poésie puisqu' il parle de vide, d'absence, de douleur et de solitude. Il n'est pas au-delà de tous les concepts métaphysiques nommés et se retrouve dans une interrogation persistante qui est peut-être sa réponse. Il vit sa nuit en espérant que l'aube sera plus clairvoyante mais il ne semble pas toujours y croire quand il écrit :

Sur la nuit

La parole que tu poses:

Miette emportée par le flot

Dans cet espace de silence et de nuit une présence est espérée et le poète la quémande fiévreusement. Il écrit "Faillir à toute présencelpar plus de présence ". Plus de présence au monde et au poème, toujours plus de 
rumination des mots tendus sur la page dans une brièveté qui nous est déjà connue chez Guillevic en particulier, qui nous dit lui, "Je suis un ruminant/Je broute des mots ", ce qui, chez Paul Farellier, semble également en complète adéquation avec sa façon de travailler le poème.

"Ces mots/Tu crois quils t'emportent/Ils ne sont que fragments déterrés" écrit-il. Le poète constate que les mots sont usés, qu'ils sont morts, qu'ils n'ont plus la vitalité attendue. Faut-il se livrer à un long travail d'archéologue pour remettre au jour le poème? semble-t-il s'interroger. Modestement, Paul Farellier fait comprendre qu'il va remettre au jour quelques bribes d'existant, qu'il va travailler sur des chantiers déjà explorés et tenter de réactiver une mémoire qui lui semble vouée à l'oubli. Sans doute, tout a été écrit, ou presque, et nous avons lu tous les livres, mais le poète a ses raisons de vivre le poème.

Paul Farellier reprend les mots, modèle ses poèmes avec une rigueur qui le conduit à l'essentiel de ce qu'il veut et peut livrer. Ses mots vont et viennent, vivent et meurent, sonnent l'angélus du soir plus que matines et laudes car chez lui le couchant semble plus prégnant que l'aube. Le poète appelle à la nuit et au silence, se projetant dans un espace inconnu dont il voudrait percer le mystère. Il sait que l'éternité ne peut être de la terre mais pourquoi ne serait-elle pas de l'univers? Le poète est dans un réel impalpable ce qui lui donne la force et le désir de continuer son poème.

Mais, nommons les choses par leur nom et ne nous cachons pas derrière des mots comme durée, éternité, absence, disparition. La mort est du vivant. Le poète même s'il transcende son lexique ne parvient pas toujours à nous apaiser car il est lui-même dans une inquiétude existentielle quand il écrit : "Et la durée se révèle infime » et plus loin : "Le soir, le vide est à refaire/Nous construisons les décombres".

Le monde apparaît-il si noir à Paul Farellier qu'il semble vivre une aube tardive et attendre "cette voix qui tombel loin de ta parole et de son temps"? La seule construction possible serait-elle ruines et décombres? L'éclat du soleil l'aveugle et seules les étoiles semblent pouvoir l'éclairer. Le poète continue d'appeler, de montrer, de sonder la nuit avec force dans sa beauté déchirante pour éclairer l'autre face du miroir.

Monique W. Labidoire : La parole poétique n'a-t-elle plus droit de cité sauf dans quelques chapelles? Toute espérance est-elle enfouie sous les ténèbres et n'avons-nous plus aucune chance de retrouver la parole perdue, celle qui nous ferait aimer le jour comme la nuit, la vie comme la mort, le bruit comme le silence? 
Paul Farellier : Je crois quil existe encore des nations poétiques. Des groupes humains se reconnaissent dans des poèmes : la langue chantée est leur langue; la métaphore, un monument familier, l'accomplissement du désir populaire.

Ces civilisations-là n'ont pas produit Racine, Mallarmé ou Char. Et il est de fait que la poésie française, elle, n'est pas l'affaire de la nation. La poésie française, je la vois comme une plante en pot, une plante de jardin d'hiver. Il faut la protéger, comme la langue française elle-même. Mais ne regrettons pas l'apparente faiblesse que traduit son étrange caractère de repliement individuel: chaque fois qu'elle a cherché à forcer sa nature, elle n'a produit qu'un méchant populisme littéraire. Sa vraie nature? Aristocratique (notez bien que je ne dis pas "élitiste "; aristocratique comme je l'entends, cela vaut même pour François Villon, en dépit des apparences). C'est sans doute cela qui a pour effet de rendre chez nous plus exemplaires qu'ailleurs les divorces modernes de la poésie qu'entraîne la crise généralisée du langage et de l'écriture.

Votre interrogation a quelque chose d'assez tragique. Vous évoquez une parole perdue. Je ne vois pour ma part, aussi ténébreuse que soit la condition présente, aucune raison de désespérer du verbe en poésie.

Paul Farellier ne désespère pas de la parole poétique. Au contraire, il s'aide de cette parole et parle bas afin que nous dressions l'oreille. "Cette vie à mourir » écrit-il et c'est le premier vers de son dernier recueil "Parlant bas sur ciel ". Cette vie est bien celle dont nous parle aussi Marc Alyn, qui est " un cadeau du ciel ". Pour Paul Farellier, elle semble être parfois un fardeau. Mais un fardeau qu'il va avoir la force de porter grâce à la beauté qu'il y consent. Grâce à cette acceptation que la vie n'est qu'un passage entre deux rives : la rive d'avant et la rive d'après, ces lieux inconnus reliés par un pont assez aléatoire. Entre l'avant et l'après, le poète dépose des instants de mémoire, la déclinaison des jours, l'absence de ceux qu'il a aimés et dessine en noir et blanc, en feu et en glace, toutes les saisons passées "quand» nous dit-il, « la couleur/n'en est plus qu’à chuchoter/le temps effacé ". Le temps s'efface comme les mots perdus en "syllabes de la poussière ", des syllabes qui tourbillonnent dans un rai de soleil et qu'on ne peut saisir. Le poète pourtant saisit les syllabes du poème comme les poussières d'étoiles de sa nuit éclairée.

Paul Farellier entre l'obscur et la lumière, au bord des rives qui ne pourront entrer en finitude sans la parole poétique, Paul Farellier donc, ne laissera pas disparaître dans la ténèbre la beauté du monde. Cette beauté 
revendique les deux faces du miroir : lumière et obscur. Ses poèmes agrandissent son champ d'existence, ce lieu traversé d'un chemin, agrippé de ronces où commence un murmure bas vers le ciel, qui tend à une parole plus éclairée. La lumière, aussi courte soit-elle dans l'été finissant, facilitera toujours l'union avec la terre.

Le poète, après une descente aux ténèbres qui peut parfois paraître sans retour, entre dans l'ordre du possible et s'éloigne un peu plus de l'ombre pour rejoindre, l'aube éclatante tout autant que la nuit griffée d'étoiles, s'élevant ainsi aux solstices pluriels qui nourriront cet espace-temps qu'il finira bien par saisir.

\section{Références}

L'Intempérie douce, Le Pont de l'Épée, 1984 (épuisé).

L'T̂le-cicatrice suivi de L'Invisible grandit, Le Pont de l'Épée, 1987 (épuisé). Une main si simple, Le Pont sous l'eau, 1989 (épuisé).

Où la lumière s'abrège, La Bartavelle éditeur, 1993.

A l'obscur et au vent, L'Harmattan, 1996.

Dans la nuit passante, L'Arbre à paroles, 2000.

Tes rives finir, L'Arbre à paroles, 2004.

Parlant bas sur ciel, L'Arbre à paroles, 2004.

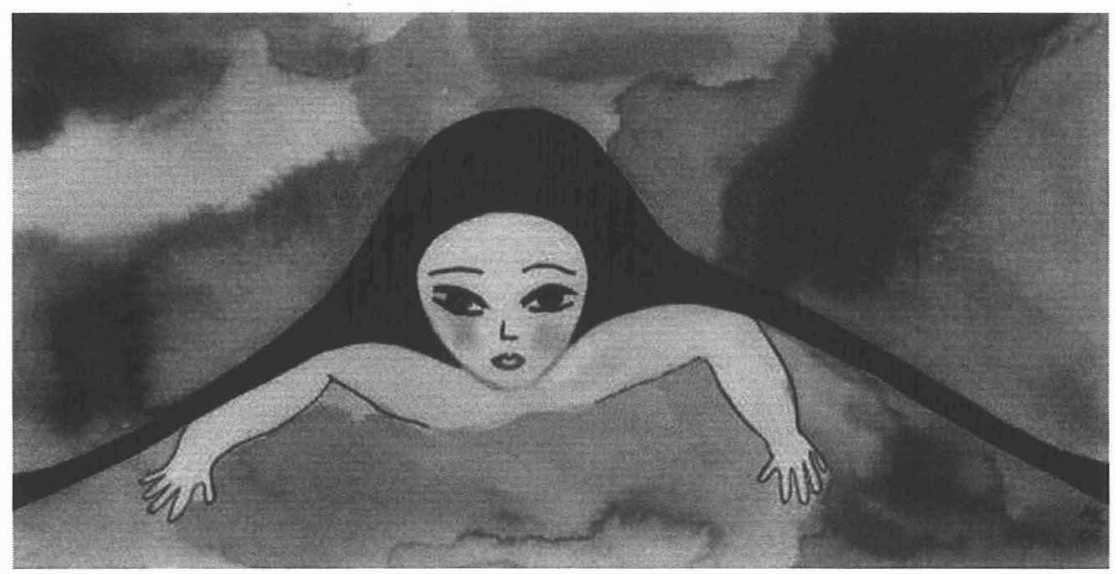

